\title{
Púrpura trombocitopénica crónica (P.T.C.)
}

\author{
Drs. Guillermo Vivanco, Mireya Bravo, Eugenia Emparanza, Jorge Vildósola y María M. Morales.
}

\begin{abstract}
ASTRACT
25 patients with C.T.P. controlled in Hospital Roberto del Rio ( 8 boys and 17 girls) ages 4 months to 13 years are presented.

Corticoid therapy was used in 23 . There was no response in 15 and in the other 8 this was dependent of its administration.

19 patients were splenectomized with $55 \%$ considered as a clinical and laboratory success, $25 \%$ of clinical success and $20 \%$ of fiulure.

From the 5 non splenectomized patients, 2 had spontas jeous remission after 8 months and 1 year respectively. Immunosuppresive therapy previous to splerectomy was performed in 3 patients without any response. In other 3 it was performed after splencx.tonty, from these in one was a slight clinical improvement but not a laboratory one, and in two there was no response at all.

Clinical symptons, laboratory masifestations and response to carticoid and inmunosuppresive therapy are analized, in addition to the evolution of the disease and the response to splenectomy. There was no conelation amony these factoes.

Therapeutic indications and their opportunities ure in arldition discussed.
\end{abstract}

De acuerdo a la duración de la sintomatología, en la Púrpura Trombocitopénica Idiopática (P.T.I.) se distinguen fornas de curso agudo $y$ crónico. ${ }^{1,2,3}$ Las formas agudas pueden presentarse como episodio único o recurrente. ${ }^{4}$ En Pediatría la forma mís frecuente es la aguda, con episo-

Servicios Departamentode Pediatriat. Hospital Robredo del Rio. Santiago. Chrile. dio único. Se presenta especialmente entre los dos y seis años de edad, con manifestaciones hemorrágicas importantes, evoluciona en forma espontinea a la curación con remisión completa y mantenida en un periodo máximo de 6 meses desde el comienzo de la enfermedad. En relación a esta fomna clínica no hay problemas diagnósticos $y$ hay consenso en cuanto a su concepto $y$ manejo.

En la forma crónica en cambio, hay grandes divergencias de opinión. De partida para algunos 
tendría características clínicas especiales como son: comienzo insidioso, manifestaciones hemorrágicas de poca intensidad, presentación preferente en niños mayores, máxima frecuencia entre los 10 y 30 años de edad, predominio del sexo femenino y falta de remisión dentro de los primeros 6 meses de enfermedad.

Sin embargo, para otros las cosas no serían tan claras y esta definición de agudo y crónico no sería tan fácil de efectuar. ${ }^{5}$

Con respecto al tratamiento, también hay grandes divergencias, especialmente en Pediatria, ya que se han descrito enfermos diagnosticados como Púrpura Trombocitopénica Crónica (P.T.C.) que han evolucionado por períodos de hasta tres años y medio y que han ide a la curación espontánea como las fonnas agudas sin necesidad de esplenectomía. ${ }^{6}$ Con esta experiencia, la indicación de Esplenectomía, de sí difícil en los niños, por los riesgos de Sepsis secundarios, es aún más difícil de tomar.

Hemos creído importante revisar la experiencia acumulada en relación a la P.T.C. en el Hospital Roberto del Río en los últimos 15 años (19651979) con el fîn de analizar tanto las fomas clinicas como los problemas terapéuticos. De este análisis se desprende que bajo el Diagnóstico de P.T.C. se incluyen formas clínicas diferentes, que merecen una cuidadosa caracterización tanto del punto de vista clínico como de laboratorio. Una vez que esto sea posible, se podrá racionalizar el manejo terapéutico y solucionar el problema que constituyen los pacientes actualmente refractarios a los tratamientos en uso.

\section{PACIENTES Y METODO}

Nuestra experiencia clínica deriva de 27 pacientes analizados retrospectivamente y que representan la totalidad de las puipuras trombocitopénicas de evolución crónica (P.T.C.) controladas en el Hospital Roberto del Rio desde diciembré de 1965 a diciembre de 1979. Siendo éste un Centro de referencia, esta casuística da una idea de la incidencia.

\section{PACIENTES Y METODO}

Nuestra experiencia clínica deriva de 27 pacientes analizados retrospectivamente y que representan la totalidad de las púrpuras trombocitopénicas de evolución crónica (P.T.C.) controladas en el Hospital Roberto del Río desde diciembre de
1965 a diciembre de 1979. Siendo éste un Centro de referencia, esta casuística da una idea de la incidencia.

Los enfermos fueron controlados y seguidos por los autores, usando los mismos criterios diagnósticos y terapéuticos.

Se consideró como P.T.C. aquellos enfermos que cumplían con los requisitos consignados en la tabla 1. Además se consideraron como P.T.C. aquellos pacientes en que se establecía ctaramente una dependencia a los corticoides, entendiendo por tal, el ascenso recuento de plaquetas hasta cifras normales durante tratamiento y caídas con su suspensión, en por lo menos dos oportunidades.

\section{Tabla 1}

Púrpura trombocitopénica crónica

\section{DEFINICION:}

1 Trombocitopenia menor de $100 \times 10^{9} / 1 t$ unantenida jor 6 meses o más.

2 Habitualmente diatesis hemorragica (petequias. equimosis y sangramiento de mucosasi.

3 Nielogratia normal (megacariocitos aumentados o normales).

4 Ausencia de un trastono primario.

5 Ausencia de esplenomegalia.

\section{Tabla 2}

Púrpura trombocitopénica crónica elementos terapéuticos usados

1 Corticoides

2 Esplenectomia

3 Inmunosupresores

4 Concentrado de Plaquetas

El estudio de laboratorio fue realizado en el Laboratorio de Hematología del Hospital Roberto del Río. El recuento de plaquetas se hizo en cámara y se controlo con la observación del frotis de sangre periférica.

Los elementos terapéuticos usados en el manejo de la P.T.C. están en la tabla 2. Los corticoides fueron usados en aquellos pacientes con trombocitopenia y manifestaciones hemorrágicas severas y en aquellos que presentaron trombocitopenia prolongada y más de 1 mes con manifestaciones hemorrágicas tabla 3 . La dosis fue de $1-2 \mathrm{mg}$ de 
Prednisona por kg de peso. No se usaron corticoides en aquellos pacientes con trombocitopenia sin maniféstaciones hemorrágicas importantes, en este grupo sólo se siguió control médico permanente.

La esplenectomía se planteó tomando en cuenta las condiciones siguientes:

- Gravedad e intensidad de la sintomatología (Hemorragia cerebral).

Tabla 3

Corticoides en PTCr

Tromlocitopenia y manilestaciones hemorrágicas severas.

INDICADO

25 PACIENTES

Trombocitopenia prolongada más de 1 mes con manilestaciones hemorrăgicas.

NO INDICADO Trombocitopenia sin manifestacio2 PACIENTES nes hemorrágicas importantes (control médico permanente). años.

- Edad. En lo posible no antes de los cuatro

- Tiempo de observación mínima 6 meses en niños mayores de 4 años.

- Imposibilidad de control hematológico adecuado después de 6 meses de evolución.

Se usaron inmunosupresores en aquellos pacientes con manifestaciones hemorrágicas importantes en quienes fall 6 el tratamiento corticoidal y esplenectomía.

Los concentrados de plaquetas se usaron sólo con las siguientes indicaciones:

- Pre-esplenectomía, inmediatamente antes, junto a otras medidas.

- Post-esplenectomía. En pacientes graves que fracasaron a la esplenectomía.

- Junto a otras medidas frente a una hemorragia cerebral.

La respuesta al tratamiento fue evaluada tanto desde el punto de vista clínico como con el recuento de plaquetas y se consideró:

Exito total: Aquellos pacientes que normalizaron su recuento de plaquetas y mejoraron clinicamente.

Exito clínico: Aquellos pacientes que solamente mejoraron ostensiblemente desde el punto de vista clínico, sin normalizar el recuento de plaquetas.

Fracaso absoluto: Aquellos pacientes en los cuales no se logró ni mejoría clínica ni del recuento de plaquetas.

\section{RESULTADOS}

La edad de comienzo y sexo de nuestros pacientes se encuentran en el gráfico 1. Queremos destacar que 3 son menores de 2 años, y 13 tienen entre 2 y 5 años. En todos los grupos etarios el porcentaje de mujeres es mayor que el de hombres, siendo de un $70 \%$ y $30 \%$, respectivamente.

PURPURA TROMBOCITOPENICO CRONICO, 27 PACIENTES. EDAD DE COUIENZO YSEX

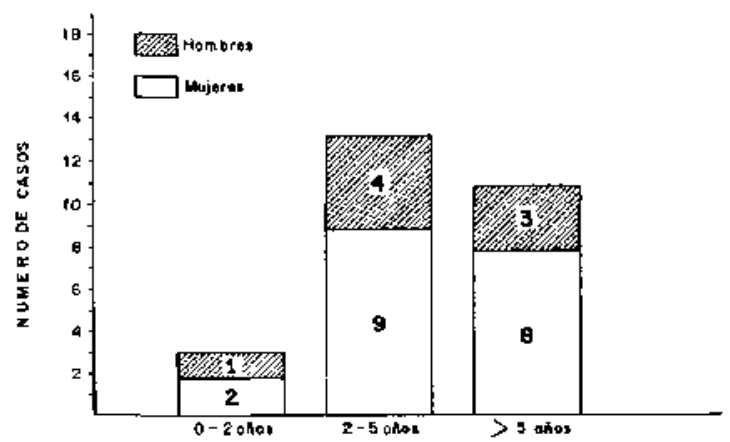

En la tabla 4 se muestra la respuesta al tratamiento con corticoides. Se trataron 25 pacientes. El recuento inicial de plaquetas por definición fue bajo en todos. Con los corticoides: 10 normalizaron su recuento y en 15 éste permanece por debajo de $100 \times 10^{9} /$. Una vez suspendido el corticaide, los recuentos volvieron a la cifra inicial en aquellos que lo habían normalizado y permanecieron en cifras comparables a lo obtenido con corticoides en los que no lo normalizaron.

\section{Tabla 4}

Corticoides en PTCr

Evolución de 25 pacientes

$\begin{array}{lll}\text { RECUENTO } & \text { RECUENTO } & \text { FECUENTO } \\ \text { PLAQUETAS } & \text { PLAQUETAS } & \text { PLAQUETAS } \\ \text { INICIAL } & \text { MAXIMOCON } & \text { DESPUES DE } \\ & \text { CORTICOIDES } & \text { SUSP. CORTI- } \\ & & \text { COIDES }\end{array}$

\begin{tabular}{ccc}
\hline $2-30 \times 10^{9} / 1$ & $250-350 \times 10^{9 / 1} / 1$ & $2-30 \times 10^{4} / 1$ \\
& $(10$ casos $)$ & $(10$ casos $)$ \\
$(25$ casos $)$ & $<100 \times 10^{9} / 1$ & $<100 \times 10^{9} /$ \\
& $(15$ cassos $)$ & $(15$ casos $)$
\end{tabular}


Se esplenectomizaron 20 pacientes tablas 5 y 6 , es decir, el $74 \%$ de los enfermos con P.T.C. De éstos, 11 pacientes ( $55 \%$ ) obtuvieron Exito total, 5 (25\%) Exito clínico y 4 (20\%) Fracaso.

El tiempo que se han controlado estos pacientes después de la esplenectomía se encuentra en la tabla 7 , aun cuando los rangos son grandes el promedio de control es de 2,4 años.

Tabla 5

Resultado de esplenectomia en 20 pacientes eon PTCr

\section{EXITO}

16

\begin{tabular}{lll} 
Recuento & Recuento & Recuento \\
Plaquetas & Plaquetas & Plaquetas \\
Nominal & Sub-normal & Bajo \\
$11(55 \%)$ & $5(25 \%)$ & $4(20 \%)$ \\
\hline
\end{tabular}

Tabla 6

Esplenectumix en 20 pacientes con PTCr (74\%)

\section{EXITOTOTAL}

(Clínico y de Iaboratorio)

EXITOCLINICO

FRACASOABSOLUTO
11 pacientes (55\%)

5 pacientes $(25 \%)$

4 pacientes (20\%)
Tabla 7

Esplenectonúa en 20 pacientes de PTCr tiempo de obsenación post operatorio

\begin{tabular}{|c|c|c|}
\hline $\begin{array}{l}\text { EXITO TOTA } \\
\text { II PACIENTE }\end{array}$ & $\begin{array}{c}\text { EXITOCLINICO } \\
\text { 5 PACIENTES }\end{array}$ & $\begin{array}{c}\text { FRACASO } \\
4 \text { PACIENTES }\end{array}$ \\
\hline & & \\
\hline
\end{tabular}

* 15-30 días (3 pac.)

* Pacientes de provincia

En la tabla 8 quisimos correlacionar el resultado de la esplenectomía con algunas características clínicas, con el fin de ver si alguna de ellas podría servir como indicador en cuanto al resultado de la esplenectomía.

Asi podemos observar que en los tres grupos no hay diferencias significativas en relación a la edad en que se presentó el primer brote purpúrico.

En relación al sexo no se pueden sacar conclusiones ya que el número de mujeres es mayor en el grupo total.

No hay diferencias en relación a tipo de comienzo, respuesta plaquetaria máxima con corticoides, presencia de bazo supernumerario ni peso del bazo.

Tabla 8

Correlación entre algunos paránetros clinicos y de laboratorio con el resuitado de la esplenectomía

\begin{tabular}{|c|c|c|c|}
\hline & EXITO TOTAL (11) & EXITO CLINICO (5) & FRACASO $(4)$ \\
\hline Edad Ier. brote & $\begin{array}{l}7 \mathrm{~m}-13 \mathrm{a} \\
(\overline{\mathrm{X}}=5.8 \mathrm{a})\end{array}$ & $\begin{array}{l}3 \mathbf{a}-9 \mathbf{a} \\
(\overline{\mathbf{X}}=4.8 \mathbf{a}\end{array}$ & $\begin{array}{l}4 a-9 a \\
(\vec{x}=6.4 a\end{array}$ \\
\hline Sexo & $\begin{array}{l}M=1 \\
F=10\end{array}$ & $\begin{array}{l}M=2 \\
F=3\end{array}$ & $\begin{array}{l}\mathbf{M}=\mathbf{3} \\
\mathbf{F}=\mathbf{2}\end{array}$ \\
\hline Tipu de comienzo & $\begin{array}{l}\text { Brusen }=10 \\
\text { Insidioso }=1\end{array}$ & $\begin{array}{l}\text { Bruscu }=5 \\
\text { Insidioso }=0\end{array}$ & $\begin{array}{l}\text { Brusco }=3 \\
\text { Insidioso }=1\end{array}$ \\
\hline $\begin{array}{l}\text { Rec. plaquetas } \\
\text { inicial }\end{array}$ & $\begin{array}{c}3 \times 10^{9} / 1=30 \times 10^{9} / 1 \\
(\bar{X}=12.3)\end{array}$ & $\begin{array}{c}8-20 \times 10^{9 / I} \\
(\bar{X}=12.6)\end{array}$ & $\begin{array}{c}6-10 \times 10^{9} / 1 \\
(\bar{X}=8.1)\end{array}$ \\
\hline $\begin{array}{l}\text { Rec. máximo con } \\
\text { Corticoides }\end{array}$ & $\begin{array}{c}206-129 \times 16^{9} / 1=3 \\
30-98 \times 10^{9} / 1=8\end{array}$ & $\begin{array}{c}130 \times 10^{9} / 1=1 \\
10-63 \times 10^{9} \pi=4\end{array}$ & $\begin{array}{c}160-200 \times 10^{9} / 1=2 \\
45-90 \times 10^{9} / 1=2\end{array}$ \\
\hline Peso del bazo (g) & $\begin{array}{l}38.2-137 \\
(\bar{X}=76.1)\end{array}$ & $\begin{array}{c}73-95 \\
(\bar{X}=80.8)\end{array}$ & $\begin{array}{c}40-197.8(\mathrm{gr}) \\
(\bar{X}=93.8)\end{array}$ \\
\hline Baza supernumerario & lenfermo & 2 enfermos & \\
\hline
\end{tabular}


Tabla 9

Correlación entre recuento máximo de plaquetas en

la primera semana y resultado de la esplenectomía

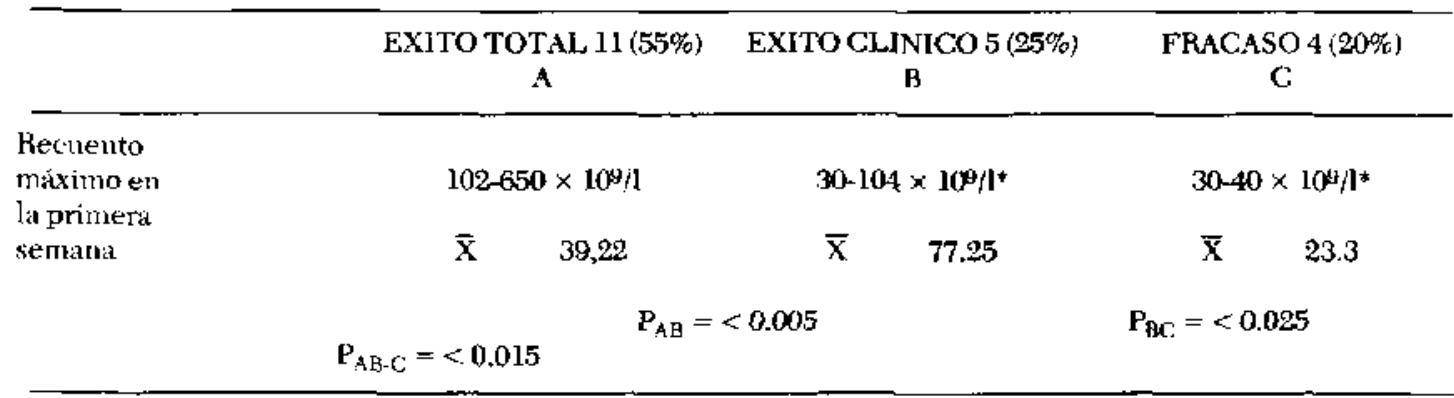

"Se eliminó 1 paciente ya que el recuento fue en relación a la administración de concentrado de plaquetus.

En la tabla 9 se ha correlacionado el resultado de esplenectomía con el recuento de plaquetas máximo dentro de la primera semana post-esplenectomía. Puede observarse que hay diferencias estadísticamente significativas entre los tres grupos.

Correlacionamos el tiempo de evolución preesplenectomía con el resultado de ella, tabla 10, y

Tabla 10

Tiempo de evolución pre esplenectomía y su relación con éxito o fracaso

$6 \mathrm{~m}-1$ año $1 \cdot 2$ años 2 años

\begin{tabular}{llll}
\hline $\begin{array}{l}\text { Fratuso } \\
\text { esplenect. }\end{array}$ & 2 & 1 & 1 \\
\hline Exito clinico & 1 & 1 & 3 \\
\hline Exito total & 2 & 3 & 5 \\
\hline
\end{tabular}

puede observarse que no hay diferencias entre los periodos observados que son entre 6 meses y dos años, es decir, que el resultado de la esplenectomía en nuestro material no tiene relación con el momento en que ésta se indico.

En la tabla 11 señalamos los riesgos de la esplenectomía, bemos tenido l meningitis neumocócica en 20 pacientes, lo que constituye un $5 \%$ de complicaciones. No hemos tenido Sepsis.

\section{Tabla 11}

Complicaciones postesplenectomía 20 casos

\section{MENINGITIS NEUMOCOCICA 0 SEPSIS $(5 \%)$}

En nuestro material hay 2 enfermos, tabla 12 , que cumplieron con los requisitos para catalogar-

\section{Tabla 12}

Evolución esponténea a la mejoria, 2 pacientes

\begin{tabular}{|c|c|c|c|c|c|c|}
\hline $\begin{array}{l}\text { EDAD } \\
\text { SEXO }\end{array}$ & $\begin{array}{l}\text { REC. PLAQ, } \\
\text { INICLAL }\end{array}$ & SINTOMAS & CORTICOIDES & REC. PLAQ. & EVOLUCION & CONTHOL \\
\hline 2 años & $14 \times 10^{9} ! 1$ & Importantes & $\begin{array}{c}1 \text { año } \\
(2 \text { curas }\rangle\end{array}$ & $\begin{array}{l}\text { Dependiente } \\
\text { del conticoide }\end{array}$ & $\begin{array}{c}\text { Subnomal } \\
1 \text { año }\end{array}$ & 4 años \\
\hline FEMI & & & & & & \\
\hline 10 años & $14 \times 10^{9 / 1}$ & Escasos & NO & & $\begin{array}{c}\text { Subnormal } \\
8 \text { meses }\end{array}$ & 1 año \\
\hline
\end{tabular}


los como P.T.C. y que despuês de 4 años y 1 año de control, respectivamente, han evolucionado en forma espontảea a la curación. Correlacionamos las características clínicas de los enfermos en que la esplenectomia fracasó, con aquellos que evolucionaron en forma espontánea a la mejoría, tabla $13, y$ puede observarse que no hay diferencias en edades, sexo, recuento plaquetario inicial; en cambio, la sintomatologia clínica fue muy diferente. Mientras aquellos pacientes en que fracasó la esplenectomía evolucionaron siempre con gran sintomatología, en aquellos que fueron a la mejoria espontánea ésta fue mínima, incluso un caso no necesitó corticoides.

Tabla 13

Correlación entre pacientes que fracasaron post-

esplenectomía y los que mejoraron espontáneamente

\begin{tabular}{|c|c|c|c|c|c|}
\hline & EDAD & SEXO & $\begin{array}{l}\text { SINT. } \\
\text { CLINICA }\end{array}$ & $\begin{array}{l}\text { RCTO. PLAQ. } \\
\text { INICIALES }\end{array}$ & $\begin{array}{c}\text { RESPUESTA } \\
\text { AL CORTICOIDE }\end{array}$ \\
\hline $\begin{array}{l}\text { Fraciasos } \\
\text { Post-esplenec. } \\
\text { (4 pacientes) }\end{array}$ & $\begin{array}{l}-6 a \\
-9 a \\
-41 / 2 a \\
-6 a .\end{array}$ & $\begin{array}{c}M \\
M \\
F \\
F\end{array}$ & $\begin{array}{l}++ \\
+++ \\
+++ \\
+++\end{array}$ & $\begin{array}{r}6 \times 10^{9} i l \\
10 \times 10^{9} / 1 \\
10 \times 10^{9} / 1 \\
6 \times 10^{4} / 1\end{array}$ & $\begin{array}{l}\text { Dependiente } \\
\text { No responde } \\
\text { No responde } \\
\text { No responde }\end{array}$ \\
\hline $\begin{array}{l}\text { Mejoria } \\
\text { espontánea } \\
\text { (2 pacientes) }\end{array}$ & $\begin{array}{l}-2 a 4 \mathrm{~m} . \\
-10 \mathrm{a} .\end{array}$ & $F$ & + & $\begin{array}{l}14 \times 10^{4 !} ! \\
14 \times 10^{9} ! 1\end{array}$ & Dependiente \\
\hline
\end{tabular}

No encontramos asociación con Anemia hemoliftica ni con Lupus eritematoso diseminado.

Se usó Azatioprine (Imuran $\left.{ }^{\mathrm{R}}\right)$, previo a esplenectomia en 3 enfemos sin obtener resultado terapéutico.

En 2 pacientes se ha usado plaquetas incubadas con Vinblastina ${ }^{\mathrm{R}}$ según técrica descrita por Ahn y col, ${ }^{7}$ en uno no se obtuvo respuesta y en el otro las plaquetas no se modificaron, pero la sintomatologia hemorrágica ha disminuido.

\section{COMENTARIO}

Cuando se analiza la P.T.I. en un número importante de enfermos, no queda duda que se trata de un sindrome en el cual están incluidas distintas formas de la enfermedad, hecho que ha sido contemplado a partir de la diferencia clásica de P.T.I. de evolución aguda, recurrente y crónica. ${ }^{3,4.9}$

Ahora bien, si se amalizan exclusivamente las formas crónicas como es el caso de esta revisión, podemos observar que aun cuando todos los pacientes pueden incluirse bajo la denominación de P.T.C., por su evolución en un período mayor de 6 meses, muchos difieren de las descripciones clásicas.
Así la P.T.C. se ha defìnido como propia de niños mayores $;{ }^{\theta, 10}$ sin embargo, en nuestro material tenemos 3 casos en menores de 2 años y 13 entre 2 y 5 años. Si bien es cierto en Pediatría la P.T.I. de evolución aguda es la más frecuente, la edad de presentación por sí sola no permite descartar una P.T.C. ${ }^{11}$

En relación a sexo, hemos encontrado predominio de mujeres $19 / 27$ en proporción semejante al del resto de la literatura 2 a $4 / 1$. $^{8}$

Las manifestaciones clinicas observadas tampoco corresponden a lo clásicamente descrito para la P.T.C. "entermedad de comienzo insidioso y curso leve". ${ }^{10}$ Así el comienzo fue brusco en 18/20 y las manifestaciones hemorrágicas intensas en $21 / 27$.

No encontramos relación entre el número inicial de plaquetas y el curso de la enfernedad, 4 pacientes con comienzo brusco y sintomatología intensa y que por esto clínicamente podria haberse esperado que seguirian un curso agudo, fracasaron al tritamiento tanto con corticoides como a la esplenectomía. En cambio, dos pacientes cuya sintomatología fue escasa pero mantenida, evolucionaron a la mejoría espontánes después de 8 meses y $l$ año de evolución.

La mejoria espontánea, aunque de baja fre- 
cuencia en la P.T.C., no es excepcional y es un hecho que hay que tener presente frente a la decisión terapéutica. Esta evolución ha sido descrita en igual forma en la literatura. ${ }^{6}$

No encontramos relación entre el número inicial de plaquetas y el curso de la enfermedad.

En nuestro material hemos realizado la experiencia de Harrington ${ }^{12}$ en enfermos que han fracasado a la esplenectomía. Al transfundir plasma de estos enfermos a sujetos normales, hemos desencadenado trombopenias transitorias. Parece claro entonces, la naturaleza inmunológica de su Púrpura. Numerosos trabajos de la literatura han mostrado otras evidencias como son la detección dé anticuerpos antiplaquetarios a través de aglutinación plaquetaria y fijación de complemento en el suero. ${ }^{13,14,15,16,17}$

Usamos corticoides en 25/27 pacientes por presentar manifestaciones hemorrágicas importantes. En ellos sólo se logró disminuir la sintomatologia clínica. Desde el punto de vista de laboratorio se demostró dependencia a la administración de la droga en 10 casos y un alza discreta del recuento de plaquetas sin Ilegar a la nomalidad en 15, en éstos áltimos los recuentos se mantuvieron bajos después de suspendida la droga. Tabla 4.

El análisis de la respuesta terapéutica de estos pacientes a los Corticoides y los hechos ya demostrados en la literatura en relación a su mecanismo de acción sumados a los riesgos de su uso hacen importante precisar algunos puntos.

Sin duda, el rol del Corticoide en la P.T.I. permanece en controversia, ya que no hay evidencias que su uso influencia el curso, duración o pronóstico, ni que las dosis usadas inhiban significativamente la producción de Anticuerpos en el hombre. ${ }^{19}$

Entre los hechos a favor de su uso hay que destacar:

- Hay evidencias de que pueden aumentar la resistencia capilar y que reducen su permeabilidad. Además aumentan la vasoconstricción. ${ }^{19}$

-Inhiben el secuestro esplénico de plaquetas jóvenes, efectivas hemostáticamente. ${ }^{y}$

- Inhiben fagocitosis de plaquetas ligadas a anticuerpos. Al disminuir esta fagocitosis podrian prolongar la sobrevida plaquetaria y obtener por este mecanismo un aumento del recuento de plaquetas. ${ }^{20}$ Sin embargo, esto último no ha sido demostrado.

- Disminuyen los riesgos de hemomagias gra- ves que ponen en peligro la vida de los enfermos. La experiencia de la literatura ${ }^{1 / 4}$ muestra que de 35 pacientes con P.T.I., cuyas hemorragias les causaron la muerte, solo 1 estaba recibiendo Corticoides. Parecería entonces que en los enfermos con hemorragias graves los Corticoides serían de utilidad para evitar riesgo de muerte.

Sin embargo, a pesar de lo anterior, el uso de Corticoides por períodos largos, en especial en la P.T.C., plantea problemas que hay que tener presentes, entre ellos:

- Mayor susceptibilidad a las infecciones.

- Producción de Cushing y úlcera péptica.

- Retardo del crecimiento.

- Trombopenia. Hay observaciones ed la literatura que muestran que el uso de grandes dosis de corticoides pueden causar trombopenia. ${ }^{23,21}$

La respuesta a los corticoides dependerá según Karpatkin $^{8}$ de:

- La capacidad de la médula ósea de aumentar el nưmero y volumen de megacariocitos.

- El bloqueo relativo que ejerzan los Corticoides sobre el S.R.E.:

- La afinidad de los anticuerpos antiplaquetarios por las plaquetas.

En nuestra experiencia no hemos encontrado correlación entre el alza del recuento plaquetario obtenida con el uso de Corticoides y el éxito de la esplenectonía como ha sido planteado. ${ }^{8}$

En resumen, los Corticoides en P.T.C. deberían usarse en aquellos enfermos con gran sintomatologia hemorrígica, por períodos cortos, no esperando mejorar el recuento plaquetario, sino disminuir los sangramientos.

Los enfermos que generalmente se benefician con los Corticoides así usados son aquellos con Púrpura muy intensa, con manifestaciones hemorrágicas severas y recuento plaquetario menor de 10.000.

La esplenectomía es el recurso terapéutico de mayor valor para el enfermo con P.T.C., cuando las medidas generales y el uso de Corticoides no han sido efectivos. ${ }^{22}$ Con ella se beneficia alrededor del $80 \%$ de los pacientes. Es el tratamiento de elección, como medida de emergencia, cuando se sospecha una hemorragia intracraneara. ${ }^{23}$

En nuestra experiencia el $55 \%$ de los pacientes obtuvo éxito clínico y de laboratorio y el $25 \%$ obtuvo mejoría clinica sin normalización del recuento plaquetario (tabla 6).

EI éxito de la esplenectomía se ha atribuido a que con ella se remueve el sitio potencial de des- 
trucción de plaquetas dañadas o sensibilizadas por anticuerpos. A su vez se elimina la fuente principal de producción de anticuerpos. ${ }^{24}$

Se ha postulado que el éxito de la esplenectomía estaría en relación con la precocidad con que se realiza, ${ }_{2}^{25}$ sin embargo, en nuestro material, no encontramos esta correlación (tabla 10).

Al plantear la esplenectomía hay que tener presente algunos hechos:

A pesar del éxito terapéutico, en el $80 \%$ de los casos hay un $20 \%$ de pacientes que no se benefician. Hasta el momento no hay manera de predecir este resultado. En nuestro material sólo el recuento plaquetario en la primera semana post-esplenectomía permitió predecir el éxito. Así, el recuento plaquetario en los pacientes que respondieron fue significativamente más alto que los que tuvieron sólo éxito clínico y en éstos a su vez significativamente más altos que en los que fracasaron. Tabla 9.

- El riesgo de sepsis post esplenectomía es un hecho conocido ${ }^{26,27}$ y en grandes estadísticas éste es hasta 100 veces mayor que el de la población general. Otros en cambio no encuentran este riesgo en los casos de esplenectomía por P.T.C. ${ }^{10}$ En nuestro material tenemos $1 / 20$ pacientes que hizo meningitis neumocócica. Lo que da una frecuencia de 5\% (tabla 11).

Por otra parte, hay que tener presente la experiencia de Lani $^{6}$ que muestra que hay enfermos que evolucionan espontáneamente a la mejoria después de períodos de 2-3 años de evolución.

Entre nuestros pacientes, dos evolucionaron a la mejoria espontánea después de 1 año y 8 meses de observación y llevan 4 años y 1 año de control respectivamente después de haber nomalizado el recuento de plaquetas.

Por estas razones pensamos que para plantear la esplenectomía, en lo posible, debe esperarse hasta que el niño tenga 5 años de edad. Esto último tanto para disminuir e] riesgo de sepsis, ya que sabemos que este no desaparece, como por la posibilidad de mejoría espontánea.

En resumen, indicamos esplenectomía en aquellos pacientes con P.T.C. con sintomatologia hemorrágica importante, en lo posible después de los 5 años de edad y de 1 año de observación. Planteamos la esplenectomía antes del año de control en las siguientes situaciones:

- De emergencia, en enfermos con hemorragias graves (hemorragia intracraneana, epistaxis permanente, hemorragia digestiva, hematuria importante) ya que la experiencia ha mostrado que en ellos hay gran riesgo de muerte y responden bien a la esplenectomía aun cuando la respuesta plaquetaria sea pobre..$^{28}$

- En fonma electiva, en enfermos con manifestaciones hemomágicas que no pueden llevar control adecuado o necesitan corticoides por períodos muy largos.

Después de practicar la esplenectomía hay que advertir a los padres los riesgos de sepsis $y$ hacer profilaxis para infecciones por neumococo (vacunación y Penicilina), para disminuir los riesgos. ${ }^{10}$

El uso de inmunosupresores (tabla 14) esta basado en el hecho que en más del $80 \%$ de los pacientes con P.T.C. se han demostrado la presencia de anticuerpos antiplaquetarios.

Tabla 14

Inmunosupresores en PTCr

Fundamento

Uso

Drogus ensadias
: Eu más del $80 \%$ de los patientes con PTCr se ha demostrado la presenciade Ac. Antiplaquetarios.

Por su alta toxicidad lo indicamos en aquellos pacientes con manifestaciones clínicas importantes en quienes ha fallado el tratarrientu corticoidal y la Esplenextomía.

\author{
:- Sulfato de Vincristínia \\ Azithioprina \\ Ciclotosfamida \\ 6.M.P. \\ Clorimbulcil
}

Por su alta toxicidad está reservado a pacientes refractarios al uso de esteroides y esplenectomía. Hemos usado Azatioprine, ciclofosfamida, pla- quetas incubadas con Vinblastina, etc. La experiencia ha sido mala. No hemos tenido respuesta adecuada clínica ni de laboratorio. Igual expe- 
riencia se encuentra en otras publicaciones. ${ }^{2}$

El uso de plaquetas incubadas con Vinblastina, ? que teóricamente parece promisorio, lo hemos usado en dos enfermos. En uno de ellos en dos oportunidades. No hemos abtenido mejoria desde el punto de vista de laboratorio, sólo una discreta disminución de la sintomatologia clínica en uno de ellos. Esta experiencia es, sin embargo, limitada $y$ no podemos sacar conclusiones al respecto.

E] uso de concentrado de plaquetas sólo tiene indicación junto a otras medidas frente a un sangramiento que pone en peligro la vida del enfermo como es la hemorragia cerebral. No tiene otra indicación en el manejo de estos enfermos, ni aun en el preoperatorio, ya que la experiencia quirúrgica ha mostrado que la hemorragia intraoperatoria no es importante.

La P.T.C. sigue constituyendo un problema diagnóstico y terapéutico y sin duda. el futuro dependerá del desarrolio de técnicas capaces de detectar anticuerpos antiplaquetarios que permitan caracterizar y diferenciar mejor las formas clínicas de Púrpura y poder tomar las medidas terapéuticas correspondientes.

\section{RESUMEN}

- Se presenta la experiencia con 25 pacientes con P.T.C. controlados en el Hospital Roberto del Río, 8 hombres y 17 mujeres, cuyas edades fluctuaron entre 4 meses y 13 años.

- Se usó Corticoides en 23 , en 15 no se obtuvo respuesta y en 8 ésta fue dependiente de su administración.

- Se esplenectomizaron 19 enfermos, obteniéndose $55 \%$ de éxito clinico y de laboratorio, $25 \%$ de éxito clínico y $20 \%$ de fracaso.

- No se esplenectomizaron 5 enfemos, 2 remitieron espontáneamente después de 8 meses y 1 año de evolución, respectivamente.

- Se usaron inmunosupresores, previos a la esplenectomia en 3 enfermos sin obtener respuesta y en 3 enfermos post-esplenectomía. De éstos últimos, en uno hubo discreta mejoría clínica, pero no de laboratorio y en dos no hubo respuesta.

- Se analiza la sintomatología clínica, de laboratorio y respuesta al trakamiento con corticoides e inmunosupresores con la evolución de la enfer- medad y la respuesta a la esplenectomía, sin encontrar elementos que permitan correlacionarlos.

- Se discuten además las indicaciones terapéuticas y su oportunidad.

\section{REFERENCIAS}

'Hirsch, E. O.; Dameshek, w. "Idiopathic thrombocytopenia: Review of 89 cases with particular reference to differentation and treatment of acute (self limited) and chronic types". lntem. Med. 88: 701, 1951.

${ }^{2}$ Larruin, $C_{\text {.; }}$ Femdndez, $\boldsymbol{H}$. Púrpura trombocitopénica idiopátiça Análisis clinico y terapéutico de 151 pacientes. Rev. Méd. Ch. 107: 824, 1979.

${ }^{3}$ Aster, R. H. Plateletdisorders. Advanced in undentanding and management. Post Graduate Medicine. 55: 50, 1974.

${ }^{4}$ Dumeshe ${ }^{\prime}, W_{\text {; }}$ Ebhe, Sch.; Creenberg, $L$; Baldini, M. Hecurrent acute idiopathic thrombocitopenic purpur. N. Engl. J. Med. 269: 647, 1963.

${ }^{5}$ Thampson, R. L.; Moore, R. A.; Hess, Ch. E.; Wheby. M. S.; Leaveli, B. S. Idiopathic thrombocytopenic purpura. Areh. Jitern. Med. 130: 730, 1973.

${ }^{6}$ Lanmmi, A. T, Lovric, V. A. Idiopathic thrombocitopenic purpura: Ar epidemiologic study. J. Pediatr. 83: 3L 1973.

${ }^{7}$ Ahn, Y. S,; Bumes, J. J,; Harrington. W. J, Caver, M, L,; Smith, D. S,; Brunkill, D. E.; Pall, $L$ E. The treatment of idiopathic thrombocitopenia with vinblastine-loaded platelets. N. Engl. J. Med, 298: 1101, 1978.

${ }^{8}$ Karpatkin, S. Autoimmune thrombocytopenic peurpura Am. J. Med. Sti, 262; 127, 1971

${ }^{9}$ Simons. S. M.; Main, C. A.; Yaish, H. M. et al. Idjopatic thrombocytopenic purpura in children. J. Pediutr. 87: 16, 1975.

"Campbell, W, Mac Millan. Platelet and vascular disonders. cup. 24. Smith's Blood diseases of infancy and childhood. 4ta. Ed. the C.V. Mosby compony Saint Lowis, 1978.

${ }^{11}$ Choi, S. I.; McClure, P.D. Idiopatic thrombocytopenic purpura in childhood. Can. Met. Ass. J. 97: 562, 1967.

12 Harrington, W. J.; Minnich, V,; Hollingsworth, J. W; Moore, C. V. Demonstration of a thrombacytopenic factor in the blood of patients with thrombocytopenic purpurz. J. Lab. Clin. Med. 38: 1,1951 .

${ }^{13}$ Morcassachi, $N_{4}$ Moorhead, J.; Leiking, J. F. Antiplatelet antibodies in childhood. Idiopathic thrombocytopenic purpura. Ain. J. Dis. Child. I33: 257, 1979.

${ }^{14}$ Kurpatkin, S.; Strick, N. Kanpatkin, M.H. et al. Cumulative experievce on the detection of antiplatelet antibocty in 234 patients with idiopathic thrombocytopenic purpura, systemic lupus erythematosis and other clinical disonders. Aner. J. Med. 52: 776, 1972.

${ }^{15}$ Karpatkin, S.; Siskind, G. W. In vitro detection of platelet antibody in patients with idiopathic thrombocytopenic purpura and systenic lupus erythematosus. Blood 33: 785, 1979.

16 Luiken, G. A.; McMillan, R.; Lightsey, A. L.; Gordon, D.; Zetely, S.; Schulman, 1.; Gribble, T. J, y Longmire, R. L. Platelets associated IgG in immune thrombacytopenic purpura. Blood 50: 317, 1977.

17 Harrington, W. J.; Spiague, C. C.; Minnich, V.; Moore, C. V.; Aubin, R. C.; Duboch, $R$. Inmunologie mechanisme in idiopathic an neonatal thrombocytopenic puppure. Ann. Intern. Med. 38: 433, 1953 . 
${ }^{18}$ Schulman, I. Diagnosis and treatment: Management of idiopathic thrombocytopenic purpura, Pediatrics 33:979, 1964.

19 MCClure, P. D. Idiopathic thrombocytopenic purpura in chil. dren: Should corticosteroids be given? Am. J. Dis. Child. 131 $357,1977$.

${ }^{20}$ McMillan, H.; Longmire, R. L; Tavassoli, M. et at. In vitro platelet phagocytosis by splenic leukocytes in idiopathic thrombocytopenic purpura. N. Engl. J. Med. 290: 249, 1974.

${ }^{21}$ Cohen, $P, y$ Gardner, $F$. H. The thrombocytopenic effect of sustained high dosuge prednisone therapy in thrombocytopenic purpura. New Engl. J. Med. 265: 611, 1961.

22 Simons, S. M.; Main, C. A.; Yaish, H. M. et al. Idiopathic thrombocytopesic purpura in children. 1. Pediatr. 8l: 16, 1975 .

${ }^{83}$ Humphrevs, R. P.; Hockley, A. D.; Freedman, M. H.; Saunders,
E. F. Marsagement of intracerebral hemorrhage in idiopathic thrombocytopenic purpura. J. Neurosurg. 45: 700, 1976.

${ }^{24}$ McClure, P. D. Idiopathic thrombocytopenic purpura in children: Diagnosis and managements. Pediatrics 55: 68, 1975.

25 McPherson, A. J. S.; Richmond, J. Plannet splenectomy in ITP. Br. Med. J. 1: 64, 1975.

${ }^{26}$ Eraklis, A. J.: Kevy, S. V.; Diamond, L. K et al. Hazard of overwhelming infection after splenectomy in childhood. $x$. Engl. J. Med. 276; 1225, 1967.

${ }^{27}$ Lucas, R. V.; Krivit, W. Overwhelming infection in children following splenectomy. 1. Pediatr. 57: 185, 1960.

${ }^{24}$ Zerell z, J. T.; Margin, L. W, y Lampkin, B. C. Emergency splenectomy for idiopathic thrombocytopenic purpura in children. Journal of Pediatric Sugery. 13: 243, 1978. 